\title{
A cautionary note on the reporting of genetic association study results
}

\author{
Journal of Human Genetics (2012) 57, 465-466; doi:10.1038/jhg.2012.47; published online 17 May 2012
}

We would like to point out a practice that is becoming widespread in reporting of associations. Odd ratios (OR) are commonly converted to percentages presumably for the benefit of the lay audience. Due to the substitution of odds for probabilities and the asymmetrical distribution of protective and risk ORs, this practice leads to misleading reporting. This is particularly troubling in genetic association studies where associations may be reported as a risk association with one allele of a biallelic single nucleotide polymorphism or a protective association with the other allele. These two versions of the same association are identical in magnitude, but the current practice results in reporting different effect sizes in percentages. Here, we suggest that direct conversion of ORs to percentages should be discontinued, and we also remind that there are useful transformations readily available as replacements.

Effect size in retrospective case-control association studies is customarily reported as an odds ratio (OR). ORs lie between zero and infinity, with unity indicating no association. For protective associations, the OR is between 0 and 1 . Risk associations yield an OR of greater than 1, the upper limit being infinity. Since many people think more easily in terms of probabilities than odds, ORs are often converted into percentage changes in risk, especially in press releases. It is now common practice to state a protective OR of 0.6 as $40 \%$ reduction in risk, assuming a rare disease. The reciprocal risk association with the alternative allele yields an OR of 1.67 and this would be reported as a $67 \%$ increase in risk. On the other hand, a protective association with an OR of 0.60 , and a risk association with an OR of 1.40 would be reported as $40 \%$ decrease and increase, respectively, in risk. The impression given is that these two associations in opposite directions are similar in magnitude, but actually they are not. Finally, the notion of fold-change and percentage increase are often intertwined, so $\mathrm{OR}=3.0$ is transformed to a $300 \%$ increase in risk, but $\mathrm{OR}=2.0$ to a $100 \%$ increase. In each of these scenarios, conversion of ORs to percentages leads to erroneous reports.

This odd situation derives from nature of the case-control study design, the asymmetry of the scales of protective and risk ORs, and the centering around the value 1 for neutrality. To be translated into meaningful percentages, ORs must be transformed so that risk and protective ORs lie within identical and finite scales. Percentage-based metrics are justifiable if the metric has zero value under statistical independence, maximum value unity, and minimum value minus unity independent of the marginal distributions. Such metrics are readily available. ${ }^{1}$ One of them is the Yule's Q value, which was originally described as an association coefficient. ${ }^{2}$ Yule defined $Q$ in terms of the $2 \times 2$ contingency table, equating $\mathrm{Q}$ with the difference between the number of concordant pairs and discordant pairs expressed as a percentage of the number of untied pairs in the table. In terms of the odds ratio, $\mathrm{Q}=(\mathrm{OR}-1) /(\mathrm{OR}+1)$, which transforms all ORs to $[-1,+1]$ scale with symmetry around zero. The Q value is recognized as a measure of allelic association in genetics ${ }^{3}$ but not commonly used in epidemiologic association studies. When this transformation is used as the measure of association as suggested by Yule, $\mathrm{OR}=1.4$ represents not $40 \%$ increase in risk, but $16.7 \%$ departure from the null relationship. Fifty percent departure is conferred by an association with $\mathrm{OR}=3.0$.

Table 1 Odds ratios and their transformed values

\begin{tabular}{lllll}
\hline $\begin{array}{l}\text { Odds } \\
\text { ratio }\end{array}$ & $\begin{array}{l}\text { Simple conversion to } \\
\text { percentages }\end{array}$ & $\begin{array}{c}\text { Yule's } Q \\
\text { transformation }\end{array}$ & $\begin{array}{l}\text { Conversion to percentages } \\
(\text { Yule's Q) }\end{array}$ & $\begin{array}{c}\text { log10 } \\
\text { transformation }\end{array}$ \\
\hline 0.0 & $100 \%$ decrease & -1.0 & $100 \%$ departure & - Infinity \\
0.001 & $99.9 \%$ decrease & -0.998 & $99.9 \%$ departure & -3 \\
0.01 & $99 \%$ decrease & -0.980 & $98 \%$ departure & -2 \\
0.25 & $75 \%$ decrease & -0.60 & $60 \%$ departure & -0.602 \\
0.50 & $50 \%$ decrease & -0.333 & $33.3 \%$ departure & -0.301 \\
0.75 & $25 \%$ decrease & -0.143 & $14.3 \%$ departure & -0.125 \\
1.0 & No change & 0.0 & No change & 0.0 \\
1.25 & $25 \%$ increase & 0.111 & $11.1 \%$ departure & 0.097 \\
1.50 & $50 \%$ increase & 0.20 & $20 \%$ departure & 0.176 \\
1.75 & $75 \%$ increase & 0.273 & $27.3 \%$ departure & 0.230 \\
2.00 & $100 \%$ increase & 0.333 & $33.3 \%$ departure & 0.301 \\
4.00 & $>100 \%$ increase & 0.600 & $60 \%$ departure & 0.602 \\
10.0 & $>100 \%$ increase & 0.818 & $81.8 \%$ departure & 1 \\
100.0 & $>100 \%$ increase & 0.980 & $98 \%$ departure & 2 \\
1000.0 & $>100 \%$ increase & 0.998 & $99.8 \%$ departure & 3 \\
Infinity & Infinity\% increase & $\sim 1.0$ & $\sim 100 \%$ departure & Infinity \\
\hline
\end{tabular}

${ }^{\mathrm{a} Q}=(\mathrm{OR}-1) /(\mathrm{OR}+1)$. 
Most importantly, ORs identical in their effect size (like 0.5 and 2.0) are equidistant from 0 when transformed to Yule's Q values very much like the log-transformed ORs (Table 1). Although both Yule's transformation and log-transformation convert risk and protective ORs equal in effect sizes to values equidistant from neutrality, the advantage of the Yule's $Q$ is that it lies between -1 and 0 (protection) or 0 and +1 (risk). This allows one to interpret Yule's $Q$ as a percent departure from neutrality, while no such intuitive interpretation is possible for the log-transformed OR. In reporting percent departure from neutrality rather than percent change in risk, both protective and risk ORs should be subject to Yule's transformation to maintain equality of their scales.

While there are some substitutes for OR in certain contexts, ${ }^{4}$ no ideal solution exists for making case-control study ORs intuitively meaningful. Despite the absence of a perfect alternative, the current practice of simple conversion of ORs to percentages needs to be discontinued. Instead, ORs should be expressed as fold-change of risk or be transformed by Yule's formula to express percentage departure from neutrality. The sampling distribution and the standard error of the $\mathrm{Q}$ statistic are easily derived from those of the log-transformed OR, so inferences and estimations can be made in terms of the new metric.

It is important to inform the public using correct figures and not to mislead the readers of journals reporting association studies. In any case, Yule's Q is a more sensible parameter than OR for routine use in association studies. By recalling the existence of a century-old simple concept, we hope that the erroneous practice in reporting of association studies will be discontinued, and authors will provide clearer reporting association study results.
M Tevfik Dorak ${ }^{1}$ and William J Amadio ${ }^{2}$

${ }^{1}$ Department of Environmental and Occupational Health, Robert Stempel College of Public Health and Social Work, Florida International University, Miami,

FL, USA and ${ }^{2}$ Rider University, College of Business Administration, Computer Information Systems Program, Lawrenceville, NJ, USA E-mail:mdorak@fiu.edu.

1 Warrens, MJ. On association coefficients for $2 \times 2$ tables and properties that do not depend on the marginal distributions. Psychometrika 73, 777-789 (2008).

2 Yule, G. U. On the association of attributes in statistics. Philos. Transact. Royal. Soc. A 194, 257-319 (1900).

3 Devlin, B. \& Risch, N. A comparison of linkage disequilibrium measures for fine-scale mapping. Genomics 29, 311-322 (1995).

4 Bjerre, L. M. \& LeLorier, J. Expressing the magnitude of adverse effects in case-control studies: "the number of patients needed to be treated for one additional patient to be harmed". B.M.J. 320, 503-506 (2000). 